\title{
An adaptive interaction architecture for collaborative GIS
}

\author{
M. Petit ${ }^{\mathrm{a}, *}$ C. Ray $^{\mathrm{a}}$ C. Claramunt ${ }^{\mathrm{a}}$ \\ ${ }^{a}$ Naval Academy Research Institute, 29240, Brest Naval BP 600, France
}

\begin{abstract}
The research presented in this paper explores and models the interactions needed for the development of a collaborative and adaptive Geographical Information System (GIS). The proposed framework is based on a multi-dimensional contextual approach that makes the difference between the user, geographical and device contexts. The spatial distribution of the GIS functional components allows for the characterisation of different geographical context configurations. These configurations act as a support for the derivation of user groups. The interfaces and functionalities offered by the adaptive GIS are modeled at the group level, and derived from the interface usages and habits. The spatial behaviours that denote user experiences within a group are shared with other users within that group. Such an approach provides an adaptive and collaborative environment that favours exchanges and enrichments of the quality of services offered to the users acting in such a GIS environment. The framework is applied and experimented in the context of maritime navigation.
\end{abstract}

Key words: Adaptive and mobile GIS, collaborative filtering, context-awareness

\section{Introduction}

The research presented in this paper introduces a context-aware mobile GIS that integrates adaptive interaction principles. We define an adaptive GIS as a generic GIS that can be automatically updated according to several contexts defined by (1) the properties and location of the geographical data manipulated, (2) the underlying categories that reflect different group profiles and (3) the characteristics of the computing systems and supporting web and

\footnotetext{
* Corresponding author.

Email addresses: petit@ecole-navale.fr (M. Petit), ray@ecole-navale.fr

(C. Ray), claramunt@ecole-navale.fr (C. Claramunt).
} 
wireless techniques. This classification has been inspired by a related work introduced by Calvary et al. (2003). These contexts correspond to the components that influence, to a certain degree, the diffusion of geographical data in wireless environments. The dimensions identified are of different nature as they involve geographical data, computing processes and interfaces, and user categories. These dimensions are not new when studied individually, but less considered as a whole. For instance, previous works in the field of adaptive GIS introduce a technology-driven approach for an hardware-based interaction medium (Hampe and Paelke, 2005)(Burigat and Chittaro, 2005). Adaptation of an open GIS layer descriptor to specific user needs and contexts have been also studied in (Zipf, 2005). A context-sensitive model for mobile cartography that emphasizes different levels of data adaptation and presentation have been proposed in (Reichenbacher, 2003). In the collaborative domain, a GIS is commonly used as a dialog and interaction medium between different users (Rauschert et al., 2002)(MacEachren and Cai, 2006). Implicit collaboration between several users may be used to recommend an interface content (Brown et al., 2002). These works implicitly show the diversity of the notion of context, and the need for an integrated approach of the problem.

Over the past few years the development of personalized software has been the object of considerable attention (Riecken, 2000). Web information engineering offers promising solutions for personal content profiling. For example, many differents algorithms have been tested and are currently used by e-commerce Web sites (Schafer et al., 1999). A key issue in modelling user preferences is to approximate user intentions with a few information inputs. The techniques used for extracting user preferences and categories vary from explicit user feedbacks (Shearin and Lieberman, 2001) where a software agent learns user interests by interacting with her/him; to implicit tracking of user actions where preferences are deducted from her/his actions (Yang and Claramunt, 2005). A promising direction currently explored, takes a functional point of view where users are categorized according to their behaviours (Oard and Kim, 2001). Most of these approaches are based on the fact that users are likely to share interests when they belong to a same category. A general drawback of existing methods is that no assumption can be made regarding a new user entering a profiling system. A user passes through a "cold start" period of undetermination while her/his profile is partialy defined (Schein et al., 2002). Profiles may not immediatly reflect shifts in user behaviour, leading to an inappropriate inertia of a user profile. This "profile inertia" problem was emphasized in the domain of web service personalization (Lam et al., 1996).

Maritime navigation encompasses several properties that form a relevant experimental context for the development of a collaborative and adaptive GIS: user mobility, spatial distribution of services, and variability of user needs. In a previous work, we introduced an architecture and real-time services for the diffusion of maritime geographical information, at different levels from the 
global monitoring of the maritime traffic of a given area (Desvignes et al., 2002), to individual services on request (Dubs and Kaufmann, 2006).

The objective of the research presented in this paper is to consider the environmental knowledge of a distributed GIS as the input of a collaborative adaptation process that avoids the "cold start" and the "profile inertia" problems. We consider the way a given user acts in the environment as a contextual input of an adaptive GIS. The characterisation of user behaviours and aggregation of users into groups of similar properties aim to lower the learning process of novel users. Proposed interfaces and contents are adapted to a given situation, and shifts in behaviours and data demands are reflected by a regular derivation of profiles within each group.

The remainder of this paper introduces a modelling approach applied to distributed GIS, and experiments it in the context of maritime navigation, an emerging field of GIS that combines mobility and distributed services. Section 2 presents a framework for the integration of contextual dimensions into a collaborative GIS. Section 3 introduces and models the notion of geographical context. Section 4 describes the principles that underline the concepts of user groups, and their derivation mechanisms. Section 5 introduces collaborative interactions at the group level, and how these serve as a support for a generation of adaptation profiles. Finally section 6 concludes the paper and draws some perspectives.

\section{Adaptive GIS framework}

The collaborative component of the adaptive GIS framework is derived from user behaviours and patterns that allow for the generation of different users groups. The computing and interface environment considered are the one of a mobile and distributed GIS, considered as an adaptive GIS.

We characterise such an adaptive GIS by three contextual dimensions (Fig. 1). An adaptive GIS should be able to automatically derive its content and interface from a changing environment. This implies to present relevant information to the user, and to improve the usage and usability of the information provided. An adaptive GIS is built upon several services that integrate geographical data and services. Our framework considers that an adaptive GIS is built on one-to-many location based services, preferably bounded by a common user interface and that delivers geographical data, visualization and querying facilities. The context of a mobile GIS, with respect to a given user acting in the environment, varies according to the status of the different contextual dimensions considered. Facilities offered by a service may be available or not regarding the current geographical context (i.e. what and where). Available 
facilities can be refined by taking into account user preferences (i.e. who) and appliances capabilities (i.e. how). These contexts form the core of an adaptive GIS, they are defined as follows:

- The geographical context identifies to which degree services are available according to the user location and the spatial distribution of the service components (Petit et al., 2006).

- The appliance context specifies the interaction interfaces of the computing system available to a given user. These interfaces allow the user to visualize and interact with GIS data. Appliances are organized into classes of similar capabilities.

- The user context objective is to sort users into groups of similar behaviour over time and space with respect to a given service. The user context qualifies the adaptation process based on the common behaviours of the users within a group.

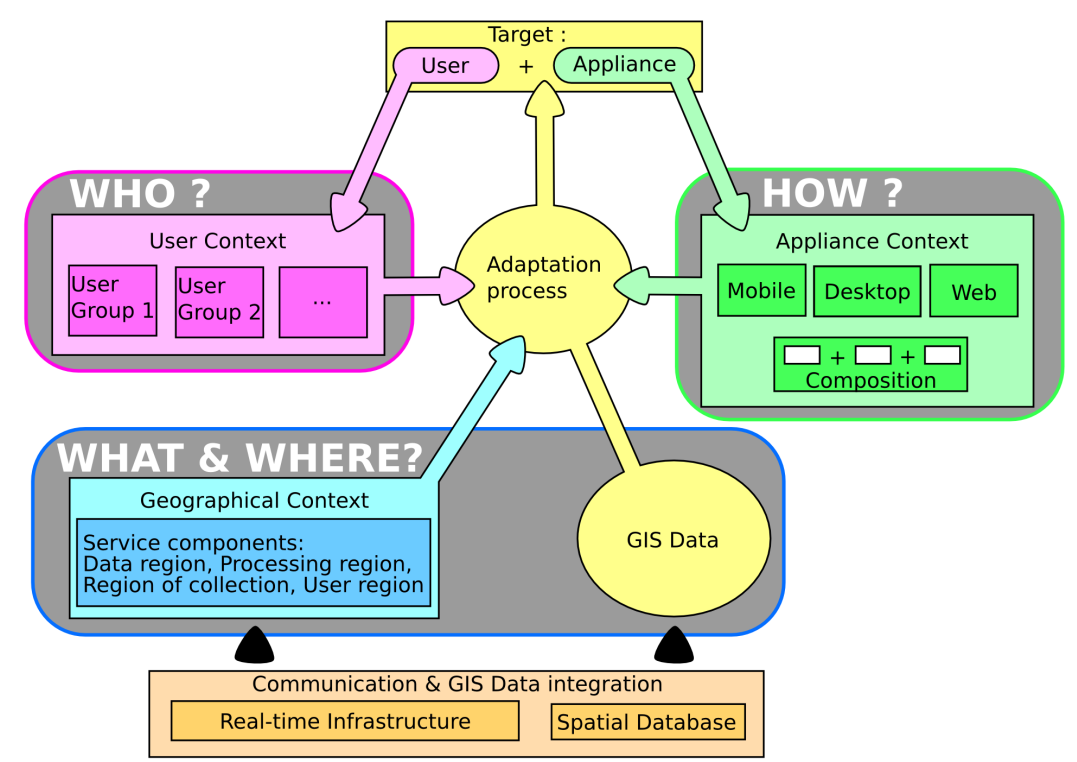

Fig. 1. Context-aware architecture of an adaptive GIS

The objective of the adaptation process is to concatenate the constraints associated to each context, and adapt the GIS services requested for a specific user and appliance by adapting both the contents and containers of the interface. This adaptive GIS constitutes an intermediate layer between data integration and presentation. From a computing point of view, geographical data are delivered by a generic communication and integration layer whose role is to aggregate different data flows from either real-time infrastructures, or previously stored geographical information that can be of interest to the end-user. This layer relies on either a wired/wireless network or a spatio-temporal database or a combination of these resources. 


\section{Geographical context}

An adaptive GIS offers interaction facilities to the users through a set $S E R$ of services. For a given service $i \in S E R$, geographical data are presented to the user by an adapted view. Unlike a conventional GIS which is usually integrated within the user desktop; in a distributed and mobile environment, an adaptive GIS is highly dependent on the services availability, particularly the ones that provide the views derived from the geographical data. Moreover, the components constituting a service are distributed over space. Based on the definition of a distributed GIS given by Goodchild in (Longley et al., 2005), we introduce several regions of significance to characterize the geographical context associated to a given service $i$, accessed by a given user $j$ at a given time $t$ (Petit et al., 2006).

\section{Definition 1 (Regions of signifiance)}

Let us consider a service $i$ and a user $j$ at a given time instant $t \in T, T$ being the set of time instants defined as reals, the regions of signifiance are defined as follows:

- $U_{i, j, t}$ denotes the region of the user $j$ and its appliance from where she/he would like to obtain GIS-based information from the service $i$ at the time instant $t$

- $S_{i, j, t}$ denotes the region of collection where geographical data of the service $i$ at the time instant $t$ are collected from

- $D_{i, j, t}$ denotes the region where the geographical data from the region $S_{i, j, t}$ is accessible to the service $i$ at the time instant $t$

- $P_{i, j, t}$ denotes the region of the service $i$ at time instant $t$ from where the data coming from $D_{i, j, t}$ are processed and broadcasted into a view, allowing the user $j$ to interact with the data

These regions constitute a multi-layered space that varies in time and whose configurations impact the availability of a given service. More precisely, for a given service and user, and at a given instant of time, we say that the set of pairs of regions of significance that intersect constitute an instant-based geographical context.

Definition 2 (Instant-based geographical context configuration)

Let $R$ denote the set of regions of significance for a given user $j$ and service $i$ at a time instant $t \in T, R=\left\{U_{i, j, t}, D_{i, j, t}, P_{i, j, t}, S_{i, j, t}\right\}$. An instant-based geographical context $g c[i, j, t]$ is given by

$$
g c[i, j, t]=\left\{\left(r_{k}, r_{l}\right) / r_{k}, r_{l} \in R \wedge r_{k} \neq r_{l}, r_{k} \cap r_{l} \neq \emptyset\right\}
$$


A geographical context may also hold over an interval of time. We define an interval-based geographical context as follows.

\section{Definition 3 (Interval-based geographical context configuration)}

Let $\left[t_{s}, t_{e}\right]$ denote an interval of time where $t_{s}, t_{e} \in T$ are time instants with $t_{e}>t_{s}$. We say that a geographical configuration for a given service $i$ and user $j$ holds over a time interval $\left[t_{s}, t_{e}\right]$ when it holds over all the time instants of that interval. More formally

$$
g c\left[i, j,\left[t_{s}, t_{e}\right]\right] \Leftrightarrow \forall t_{a}, t_{b} \in\left[t_{s}, t_{e}\right], g c\left[i, j, t_{a}\right]=g c\left[i, j, t_{b}\right]
$$

From the interval-based definition of a geographical context configuration, the next step concerns the identification of the maximum temporal extent of a given geographical context. We then define a maximum interval-based geographical context as an interval-based geographical configuration that does not hold just before or just after its interval.

Definition 4 (Maximum interval-based geographical context configuration) Let $\mathcal{F} G c m a x\left(i, j,\left[t_{s}, t_{e}\right]\right)$ be a Boolean function that returns 1 when the interval $\left[t_{s}, t_{e}\right]$ denotes the maximum temporal extent of the geographical context represented or 0 otherwise. More Formally

$$
\begin{aligned}
& \mathcal{F} \operatorname{Gcmax}\left(i, j,\left[t_{s}, t_{e}\right]\right)= \\
& \left\{\begin{array}{l}
1 \text { iff }\left(\forall t_{a} \in T, t_{a}<t_{s} \exists t_{b} /\left(t_{a}<t_{b}<t_{s} \wedge g c\left[i, j, t_{b}\right] \neq g c\left[i, j, t_{s}\right]\right)\right) \\
\wedge\left(\forall t_{c} \in T, t_{e}<t_{c} \exists t_{d} /\left(t_{e}<t_{d}<t_{c} \wedge g c\left[i, j, t_{d}\right] \neq g c\left[i, j, t_{e}\right]\right)\right) \\
0 \text { otherwise }
\end{array}\right.
\end{aligned}
$$

The scenario illustrated in Fig. 2 shows a geographical environment where a ground station collects and broadcasts the data to a client ship. These data provide basic topographic information on the island coastline hereafter denoted as the region $S_{s_{1}, u_{1}, t_{0}}$. The ship that processes geographical data cannot communicate with either the ground station or the client, but communication between the client and the ground station is available (Fig. 2(a)). Regarding the geographical context (Fig. 2(b)), each time a wireless transmission can be established between the computing system that supports two given regions of signifiance, an intersection between the considered region should occurs at the geographical context level. This configuration is characterised as $g c\left[s_{1}, u_{1}, t_{0}\right]=\left\{\left(U_{s_{1}, u_{1}, t_{0}}, D_{s_{1}, u_{1}, t_{0}}\right),\left(U_{s_{1}, u_{1}, t_{0}}, S_{s_{1}, u_{1}, t_{0}}\right),\left(P_{s_{1}, u_{1}, t_{0}}, S_{s_{1}, u_{1}, t_{0}}\right)\right\}$. As the processing region $P_{s_{1}, u_{1}, t_{0}}$ does not intersect neither $D_{s_{1}, u_{1}, t_{0}}$ nor $U_{s_{1}, u_{1}, t_{0}}$, the resulting view delivered by the service $s_{1}$ cannot be made available to the 
user $u_{1}$ located at $U_{s_{1}, u_{1}, t_{0}}$. Fig. 2(b) also introduces a tabular notation to summarize the relationships that constitute the context configuration $g c\left[s_{1}, u_{1}, t_{0}\right]$. A black cell denotes a non empty intersection between two regions of significance, while a white cell denotes an empty one.

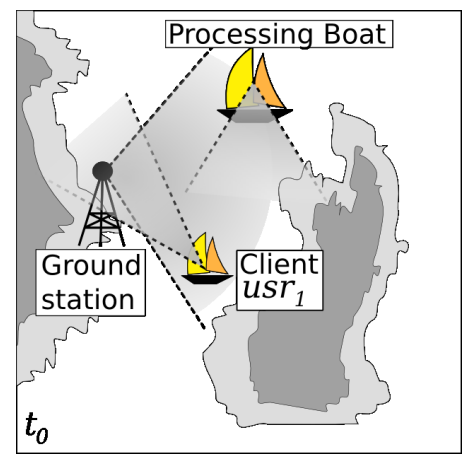

(a) Communication infrastructure view

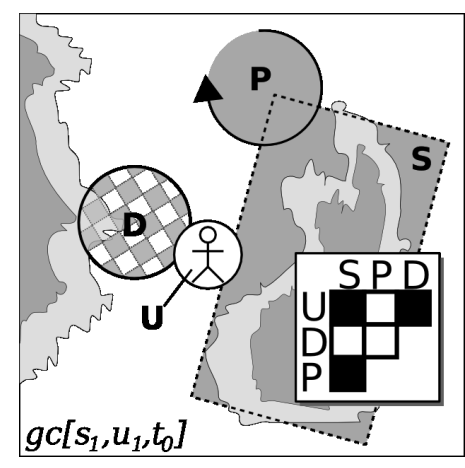

(b) Service $s_{1}$ components for the user $u_{1}$

Fig. 2. Geographical context characterization

The available service functionalities offered to a given user $j$ at a given time $t$ are inferred from the context configuration between a service $i$ and that user $j$. All functionalities of the service $i$ are fully available when $\left\{\left(U_{i, j, t}, P_{i, j, t}\right)\right.$, $\left.\left(P_{i, j, t}, D_{i, j, t}\right)\right\} \subseteq g c[i, j, t]$. This represents the case where the service $i$ is able to process a data source from $D_{i, j, t}$ at $t$ and to present a geographical data view and tools to the user $j$. Geographical configurations that do not fullfill this constraint does not denote a context rich enough of the service to construct a complete view and thus restrict the range of functionalities presented to the user. As the regions of signifiance that together characterize a service situation may be mobile over space, the context configurations are likely to evolve over time, and so are the functionalities at disposal.

Let us mention some of the functionalities that can be derived from a given restricted context. Considering a service $i$ and a user $j$, at a given time $t$, when $U_{i, j, t}$ intersects $D_{i, j, t}$, even with no processing available, the data can be stored on the user appliance for a possible and future replay into a view whenever $U_{i, j, t}$ comes to intersect the processing region $P_{i, j, t}$. When $U_{i, j, t}$ intersects $S_{i, j, t}$ and $P_{i, j, t}$, considered user is collecting data and acting in the service region of collection. At that stage the user may be allowed to input or modify data produced.

Let us consider the example presented in Fig.2, the region $P_{s_{1}, u_{1}, t_{0}}$, associated to the service ship, gets closer to $U_{s_{1}, u_{1}, t_{0}}$ and $D_{s_{1}, u_{1}, t_{0}}$ untill it intersects them (Fig. 3(a)). The context configuration changes from $g c\left[s_{1}, u_{1},\left[t_{0}, t_{1}\right]\right]$ to $g c\left[s_{1}, u_{1},\left[t_{4}, t_{5}\right]\right]$ passing through different geographical contexts. From $t_{0}$, the user $u_{1}$ located at $U_{s_{1}, u_{1}, t_{0}}$ can receive and store data diffused in the region 
$D_{s_{1}, u_{1}, t_{0}}$. From $t_{2}$, the regions $D_{s_{1}, u_{1}, t_{2}}$ and $P_{s_{1}, u_{1}, t_{2}}$ can communicate and the information on the location of the processing region is given to the user. Finally, at $t_{4}$, the user reaches the processing area, and the view associated to the service is displayed on her/his appliance. This evolution of context configurations is associated to the corresponding evolution of the functionalities offered to the user. When the geographical context changes, the available functionalities are added to or removed from the appliance interface (Fig. 3(b)).

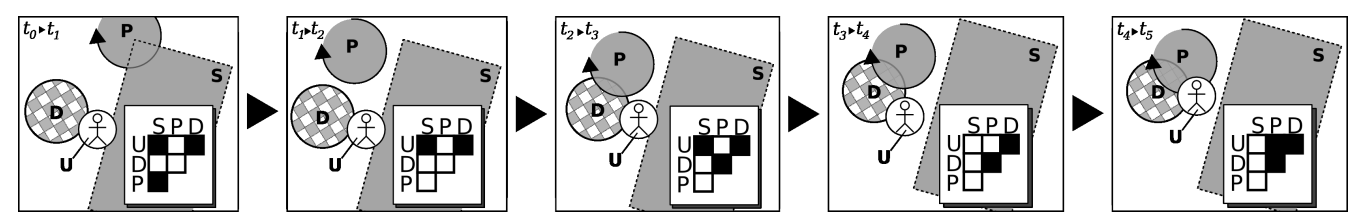

(a) Geographical context evolution
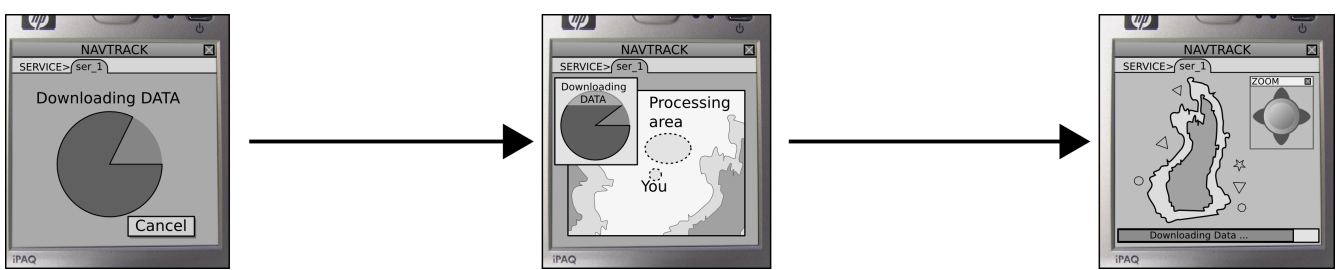

(b) Adaptive interface changes

Fig. 3. Geographical context and related interface evolution

The time-intervals associated to the successive maximum interval-based geographical context configurations encountered by a user regarding a service are added to a set hereafter denoted as a sequence of geographic context configurations.

\section{Definition 5 (Sequence of geographical context configurations)}

Let us consider a service $i$, a user $j$ and a temporal interval $\left[t_{s}, t_{e}\right]$. A sequence $\operatorname{seq}\left[i, j,\left[t_{s}, t_{e}\right]\right]$ denotes the set of time intervals of maximum interval-based geographic context configuration valid within the time interval $\left[t_{s}, t_{e}\right]$. More formally

$$
\operatorname{seq}\left[i, j,\left[t_{s}, t_{e}\right]\right]=\left\{\begin{array}{l}
{\left[t_{a}, t_{b}\right] /\left[t_{a}, t_{b}\right] \subseteq\left[t_{s}, t_{e}\right]} \\
\wedge \mathcal{F} \operatorname{Gcmax}\left(i, j,\left[t_{a}, t_{b}\right]\right)=1
\end{array}\right\}
$$

The sequence $\operatorname{seq}\left[i, j,\left[t_{s}, t_{e}\right]\right]$ represents the time-ordered evolution of context configurations from a time instant $t_{s}$, when the sequence is created, to a time instant $t_{e}$. Fig. 4 shows a graphical view of sequence $\operatorname{seq}\left[s_{1}, u_{1},\left[t_{0}, t_{5}\right]\right]$ and the associated maximum interval-based geographical context that illustrates the contextual changes examplified by Fig. 3(a). As for the regions of signifiance and context configurations, sequences are tied to a unique service. They 


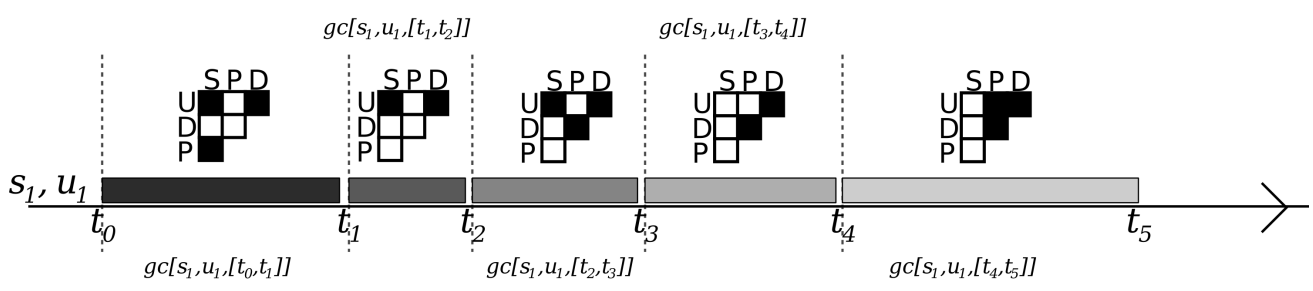

Fig. 4. Graphical view of a sequence

constitute the background behaviour of a user regarding the geographical distribution of the service components. Sequences are considered as parts of the user's profile and are stored on the user appliance.

\section{User group modelling}

A geographical context allows for the characterization of the functionalities of a service that have to be made available to the user. On the other hand, the objective of the user context is to derive and provide an adaptation process with the constraints that these should refine the content and the user interface of the functionalities provided. The adaptive GIS framework allows for multiple users to simultaneously access the functionalities offered by a given service. Let us consider several users $u_{1}, u_{2}, \ldots, u_{n}$ acting in the environment at a given instant time $t$ and interacting with a given service $i$. The set of these users with respect to the service $i$ is denoted as $U S R[i, t]$.

User interactions within a collaboration group are considered as a way to define the user context and derive the rules of adaptation of a given service. By implicitly tracking the user intentions, and without annoying the user with unsolicited actions, common usages within a group are derived. This allows the system to adapt current available functionalities according to a collaborative consensus. Collaboration between the users is not explicit as it can be in a peer-shared software, but instead works as a background process for the user context benefits.

The problem related to derivation of the groups leads us to examine different modelling issues. The first one is the identification of the range of actions that constitute the input of the categorisation process. Categorisation also implies to define the extent of the users groups, or in other words the number of users groups that makes sense from an application point of view. Finally, the developed system should be able to integrate an algorithm that binds the users to a given group according to their behaviours.

A specific characteristic of the adaptive system developed so far is that the 
user actions on the geographical space are modelled according to sequences of geographical contexts as introduced in section 3. As these sequences denote user intentions and actions regarding a service, they constitute a potential candidate to be the primitives that constitute the input of the categorisation process.

Regions of signifiance others than the user region are service-level dependent. The users have no influence on their location or extends as these characteristics only depends on the computing architecture beneath each region, with in particular, their communication capabilities. Therefore, when considering a service $i$ and two users $j, k$, their data diffusion regions, processing regions and regions of collection are identical.

Due to the underlying communication infrastructure, information sharing between two given users $j, k$ of a service $i$ and at a given time instant $t$ can only be established when their associated regions $U_{i, j, t}$ and $U_{i, k, t}$ intersect with at least one same region of significance $D_{i, j \mid k, t}, P_{i, j \mid k, t}$ or $S_{i, j \mid k, t}{ }^{1}$.

\section{Definition 6 (Communication between two users)}

Let $\mathcal{F}_{\text {comm }}(i,(j, k), t)$ be a Boolean function that returns 1 when users $j$ and $k$ of a service $i$ share an equivalent instant-based geographical context at the time instant $t \in T$ or 0 otherwise. More formally

$$
\begin{aligned}
& \mathcal{F} \operatorname{Comm}(i,(j, k), t)= \\
& \left\{\begin{array}{c}
1 \text { iff }\left(\left(S_{i, j, t}, U_{i, j, t}\right) \in g c[i, j, t] \wedge\left(S_{i, k, t}, U_{i, k, t}\right) \in g c[i, k, t]\right) \vee \\
\left(\left(D_{i, j, t}, U_{i, j, t}\right) \in g c[i, j, t] \wedge\left(D_{i, k, t}, U_{i, k, t}\right) \in g c[i, k, t]\right) \vee \\
\left(\left(P_{i, j, t}, U_{i, j, t}\right) \in g c[i, j, t] \wedge\left(P_{i, k, t}, U_{i, k, t}\right) \in g c[i, k, t]\right) \\
0 \text { otherwise }
\end{array}\right.
\end{aligned}
$$

When for two users $j, k \in U S R[i, t], \mathcal{F} \operatorname{Comm}(i,(j, k), t)=1$, the server underneath a region $D_{i, j \mid k, t}, P_{i, j \mid k, t}$ or $S_{i, j \mid k, t}$ shared by the users $j$ and $k$ acts as a bridge for information transmission from one user to the other. In order to adress all the configurations of a distributed GIS, we consider $S_{i, j \mid k, t}$ as an active region of the geographical environment where the users find support of an hardware layer to ensure a communication.

Regarding the communication infrastructure (Fig. 5(a)) of the service $s_{1}$, communication between $u_{1}$ and $u_{2}$, who are sharing at a given time instant $t_{5}$ the same geographical context (Fig. 5(b) and Fig. 5(c)), is possible through the

1 Considering a given region of significance $r \in R$ of a service $i$ and two users $j$ and $k, r_{i, j \mid k, t}$ denotes either $r_{i, j, t}$ or $r_{i, k, t}$. 
server associated to the data diffusion at $D_{i, u_{1} \mid u_{2}, t_{5}}$. The user $u_{3}$ standing in its own configuration (Fig. 5(d)) is not able to share information with the other users.

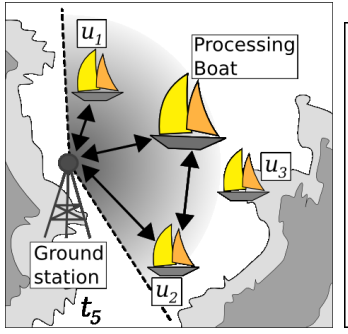

(a) Communication infrastructure view

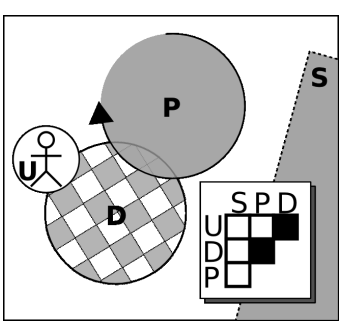

(b) Context $u_{1}$

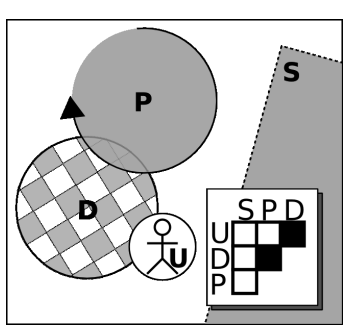

(c) Context $u_{2}$

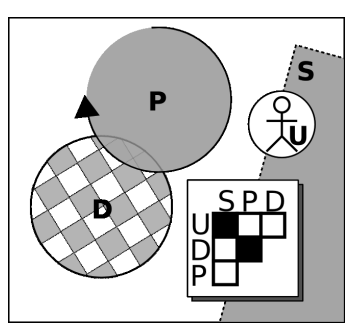

(d) Context $u_{3}$

Fig. 5. Geographical context - multiple users

As server-mediated information transmission between the users in a group is a pre-requisite to a collaborative adaptation, these groups are constituted by the users of a service that share an equivalent instant based geographical context.

\section{Definition 7 (Equivalency of instant-based geographical contexts)}

Let $\mathcal{F}$ Equiv $(i,(j, k), t)$ be a Boolean function that returns 1 when the users $j$ and $k$ of a service $i$ share an equivalent instant-based geographical context at a time instant $t$ or 0 otherwise. More formally

$$
\mathcal{F} \text { Equiv }(i,(j, k), t)=\left\{\begin{array}{l}
1 \text { iff }\left(\forall\left(r_{a}, r_{b}\right) \in g c[i, j, t], \exists\left(r_{c}, r_{d}\right) \in g c[i, k, t]\right. \\
\left./\left(r_{a}, r_{b}\right)=\left(r_{c}, r_{d}\right)\right) \wedge\left(\forall\left(r_{e}, r_{f}\right) \in g c[i, k, t],\right. \\
\left.\exists\left(r_{g}, r_{h}\right) \in g c[i, j, t] /\left(r_{e}, r_{f}\right)=\left(r_{g}, r_{h}\right)\right) \\
0 \text { otherwise }
\end{array}\right.
$$

Sharing an equivalent instant-based geographical allows the users be able to communicate as $\mathcal{F}$ Equiv returns 1 when all the regions of significance intersections inside the geographical context of a user are present in the context of the other user, and so $\mathcal{F C o m m}$ also returns 1.

We define a group as a construct that integrates the users $u_{1}, u_{2}, \ldots, u_{n}$, that share equivalents geographical context configuration at a given time instant $t$, in a set $\left\{u_{1}, u_{2}, \ldots, u_{n}\right\}$.

\section{Definition 8 (User group)}

Let us consider a given service $i$ and user $j$ at time instant $t$. A group $\operatorname{grp}[g c[i, j, t]]$ associated to this user is defined as follows

$\operatorname{grp}[\operatorname{gc}[i, j, t]]=\{k \in U S R[i, t] / \forall l \in \operatorname{grp}[g c[i, j, t]], \mathcal{F} \operatorname{Equiv}(i,(l, k), t)=1\}$ 
Let the set GRP $[i, t]$ denote the set of user groups for a service $i$ at time $t$. It is immediate to remark that a given user always belongs to no more than one group.

In the example illustrated by Fig. 5 , two groups are derived at $t_{5}$ and constitute the set $G R P\left[s_{1}, t_{5}\right]$ :

$$
\begin{aligned}
& \operatorname{grp}\left[g c\left[i, u_{1}, t_{5}\right]\right]=\operatorname{grp}\left[g c\left[i, u_{2}, t_{5}\right]\right]=\left\{u_{1}, u_{2}\right\} \text { and } \\
& \operatorname{grp}\left[g c\left[i, u_{3}, t_{5}\right]\right]=\left\{u_{3}\right\}
\end{aligned}
$$

\section{Collaborative adaptation}

At a given time, an adaptive GIS allows for a simultaneous usage of the service functionalities. Users of a service are grouped at a given time regarding their current context configuration. They store in their own profiles a sequence denoting a background view of the encountered geographical contexts with respect to this service. The scope of the collaborative adaptation is to adapt the functionalities of a given service and the user interface regarding the common behaviours of the users that belong to a same group. Mobility and contextual changes of the geographical environment are used to spread the results of the adaptation process in an ad-hoc network of servers constituted by the regions of significance.

As a user spend time in a given context configuration, she/he is likely to improve her/his usage experience of the functionalities offered. After a learning process, the user derives a deeper knowledge of the proposed user-interface and preferences regarding the geographical data she/he may request (Weakliam et al., 2005). As opposed to the case of a single user adaptive software, personal profiles are not considered as the only components of the adaptation. The groups previously defined allows for a collaborative definition of a common profile, that is, a consensus between members of a group regarding the functionalities offered by a service. Sharing the definition of an adaptation profile between the users allows for a proactive adaptation. The cold start problem for a user that becomes a group member is avoided. In that case, the new user applies the rules derived by the other users already members of in the group. The group profile encompasses the rules that materialise the common preferences and behaviour of the users member of that group regarding (1) the geographical data retrieved, (2) the functionalities offered to handle those data and (3) the user interface derived from the service $i$ at time $t$. For the remaining of this paper, we assume that prof $\left[i, g_{k}, t\right]$ designate the set of rules deriving a group profile for a given service $i$ and user group $g_{k}$ at a given time $t$. 
Whenever a standalone user or a collaborating group of users contribute to a group profile definition, an operator that aggregates and merges each of these user preferences and behavior to the group profile needs to be defined. The way this aggregation is done directly depends on the data structure implemented to qualify the user experience level. Such structures are currently available but are closely tied to a particular field of interest like web content recommending (Lieberman et al., 1999) or tourism activities (Brown et al., 2002). Within an adaptive GIS, such aggregation mechanisms and structures should be defined according to the specific properties of a given service, particularly with respect to the spatial dimension.

The group profile is an element of the set of profiles $P R O F[i, t]$, associated to the group, that is used to store possible other group profiles at a given time instant $t$. Collaborative adaptation rules are computed inside a group at a given time; the newly computed set replacing the older one. As groups are local to a given geographical context configuration, the set of group profiles are stored on the underlying servers of the regions of significance.

Let us consider a sequence of context configurations regarding a given user $j$ and service $i$. The geographical context configuration where the user spend most of her/his time denotes what we assume as the best known context. The more time a user spend in a context using the offered functionalities, the richest her/his collaboration will be regarding the collaborative rules of adaptation derived from the group associated to this context.

\section{Definition 9 (Predominant context configuration of a sequence)}

Let seq $\left[i, j,\left[t_{s}, t_{e}\right]\right]$ denote a sequence of geographical context configurations for the service $i$ and the user $j$ valid within the time interval $\left[t_{s}, t_{e}\right]$. The predominant context configuration $g c_{\text {pred }}\left[\operatorname{seq}\left[i, j,\left[t_{s}, t_{e}\right]\right]\right]$ within the sequence $\operatorname{seq}\left[i, j,\left[t_{s}, t_{e}\right]\right]$ is given by

$$
g c_{\text {pred }}\left[\operatorname{seq}\left[i, j,\left[t_{s}, t_{e}\right]\right]\right]=\left(\begin{array}{l}
g c[i, j, t] / t \in\left[t_{a}, t_{b}\right],\left[t_{a}, t_{b}\right] \in \operatorname{seq}\left[i, j,\left[t_{s}, t_{e}\right]\right] \wedge \\
\forall\left[t_{x}, t_{y}\right] \in \operatorname{seq}\left[i, j,\left[t_{s}, t_{e}\right]\right],\left(t_{b}-t_{a}\right) \geq\left(t_{y}-t_{x}\right)
\end{array}\right)
$$

In order to reflect the main contribution to the collaborative adaptation, the profile of the group where a given user collaborates most of the time is copied on her/his appliance. This predominant group profile is carried by the user along her/his sequence of context configurations.

\section{Definition 10 (Predominant group profile of a user)}

Let us consider a user $j$ using a service $i$ and her/his sequence seq $\left[i, j,\left[t_{s}, t_{e}\right]\right]$, 
her/his predominant group profile over a time interval $\left[t_{s}, t_{e}\right]$ is given by

$$
\operatorname{prof}_{\text {pred }}\left[\operatorname{seq}\left[i, j,\left[t_{s}, t_{e}\right]\right]\right]=\left(\begin{array}{l}
\operatorname{prof}\left[i, g_{k}, t_{b}\right] /\left[t_{a}, t_{b}\right] \in \operatorname{seq}\left[i, j,\left[t_{s}, t_{e}\right]\right] \wedge \\
\forall\left[t_{x}, t_{y}\right] \in \operatorname{seq}\left[i, j,\left[t_{s}, t_{e}\right]\right] \\
\left(t_{b}-t_{a}\right) \geq\left(t_{y}-t_{x}\right)
\end{array}\right)
$$

With dynamic sequences, no assumption can be made about the time spent by a user in a forthcomming context configuration. A predominant profile at $t_{1}$ may be different at $t_{2}$. In that case, the predominant profile on the user appliance is replaced by the one constructed by the group associated to the current predominant context configuration. Predominant group profiles are distributed in a peer shared network where nodes are the servers that underneath each group and users appliances are used as a mean of transportation of predominant profiles from one node to another.

The algorithm 1 (cf. appendix A) is triggered for each group $\operatorname{grp}_{k}$ of the service $i$ at a regular time and illustrates the way group profiles are derived and allows for the adaptation at the user appliance side. Three functions are used by the proposed algorithm and has to be defined according to the considered service characteristics:

- $\mathcal{F}$ AdaptTo(usr,grp_prof ): apply the adaptation rules defined in the given profile grp_prof to the user usr and its appliance.

- $\mathcal{F}$ AddCollaboration (grp_prof, usr): add the experience learned by the user usr in a group to this group profile grp_prof.

- $\mathcal{F}$ MergeProfiles $\left(\right.$ grp_prof $f_{1}$, grp_prof $\left._{2}\right)$ : returns a profile that is a combination of the rules derived in each of the two profiles grp_prof $f_{1}$, grp_prof $_{2}$ given as parameters.

The case study presented in Fig. 6 extends the previous example (Fig. 5) of three users $u s r_{1}, u s r_{2}$ and $u s r_{3}$ using a service $s e r_{1}$ and shows their evolution over the time interval $\left[t_{5}, t_{10}\right]$ with respect to three differents geographical context configurations. Fig. 6(a) denotes the geographical view of the communication infrastructure. Fig. 6(b) represents the evolution of the users sequences of geographical contexts, Fig. 6(c) displays the evolution of the user groups associated to a particular instant-based geographical context, and the set of profiles they may contain. The group profiles, member of the sets of profiles $\operatorname{PROF}\left(\operatorname{ser}_{i}, t\right), t \in\left[t_{5}, t_{10}\right]$, are represented as squares in the groups; the number of users contributing to the enrichment of each group profile of the set is also indicated. Colors filling the representations are consistent for a context, the associated group and this group profile. A step-by-step description is given to illustrate this algorithm from $t_{5}$ to $t_{10}$.

- At $t_{5}$ : Each user is alone in a group tied to one of the contexts $g c_{1}, g c_{2}$ and 
$g c_{3}$. Enrichement of the group profiles correspond to the usages of the users in the groups only. Predominant profiles copied on the user appliance are the same as the group profiles.

- At $t_{6}: u s r_{3}$ now shares the same geographical context configuration $g c_{2}$ with $u s r_{2}$. The group $g r p_{2}$ associated to $g c_{2}$ benefits from both users experiences. The predominent profile carried by $u s r_{3}$ is also copied to the set of profiles of $\operatorname{grp}_{2}$. Meanwhile, as no users remain in the group, $g r p_{3}$ is deleted.

- At $t_{7}: u s r_{1}$ and $u s r_{3}$ are in the context configuration $g c_{2}$ while $u s r_{2}$ has left that context and stays in the empty set context configuration. The predominant profile of $u s r_{1}$, that is the group $g r p_{1}$ profile is copied into the set of profiles of $\mathrm{grp}_{2}$ and $\mathrm{grp}_{1}$ is deleted.

- At $t_{8}: u s r_{1}$ and $u s r_{3}$ continue to enrich group grp $p_{2}$ profile while $u s r_{2}$ recreates a new grp $_{1}$ and carries in its predominant profile, that is, the grp $_{2}$ group profile. As the group grp $_{1}$ is derived, adaptation starts again with an empty group profile that is enriched by $u s r_{2}$.

- At $t_{9}: u s r_{1}$ switches back to $g c_{1}$ context and is associated to $g r p_{1}$. Her/his predominant profile, that comes from the $\operatorname{grp}_{1}$ existing at $t_{5}$, is merged with the new grp $_{1}$ group profile.

- At $t_{10}: u s r_{3}$ leaves $g r p_{2}$ for $\operatorname{grp}_{1}$. The copy of the group profile of $g r p_{2}$, that is the predominant profile carried by $u s r_{3}$, is merged with the one already existing in $g r p_{1}$ set of profiles. Eventually, between $t_{9}$ and $t_{10} u s r_{2}$ leaves $\operatorname{grp}_{1}$ and creates a new group grp . $_{3}$

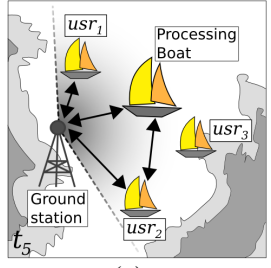

(a)

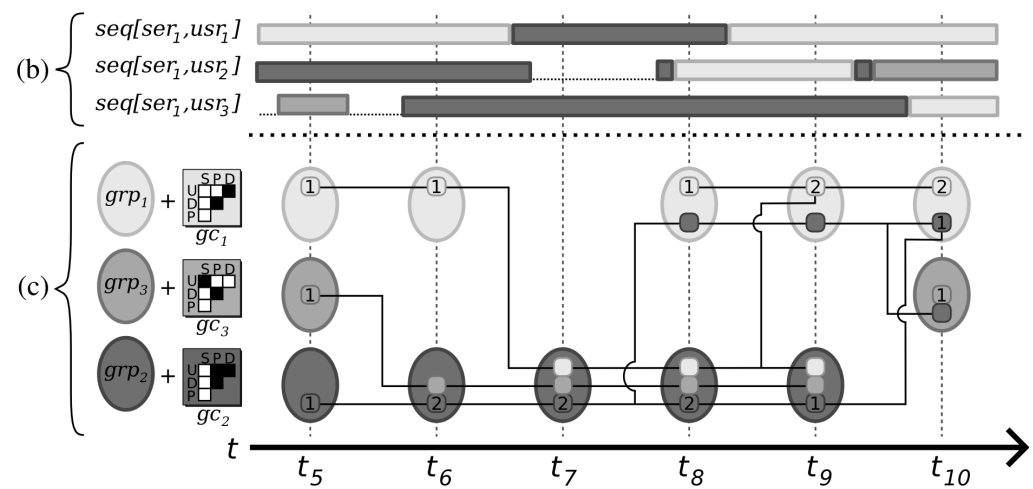

Fig. 6. User collaboration - group profiles

A prototype has been developed in order to validate the algorithm (Petit et al. 2007). This prototype simulates an evolving geographical environment of a service (Fig. 7). Users present in a given geographical environment (7(i)) and service regions of significance $(7(\mathrm{~h}))$ can be tracked in the environment (7(a)). 7(d) illustrates an example of geographical context configuration, and the group profiles related to this context. The interface of the user appliance applies adaptation rules derived from this profile. 7(e) shows the predominant context and the associated predominant profile that is stored on the user appliance. A sequence is dynamically derived when the user or service components are changing $(7(\mathrm{f})) .7(\mathrm{~g})$ shows the active groups at a current time and their 
set of profiles, including the current group profile. Currently represented user and services are changed at 7(b). and 7(c), respectively. The experience level reflected in the group profiles shows the time a user spend in a given group. The merging profile algorithm merges both experience level values into a new profile.

Preliminary experiments concern the simulation of the example illustrated in Fig. 5 The adaptive GIS is made of a service that can be accessed by several users. Users and regions intersect and users sharing a similar context configuration allow profiles to be enriched and diffused. Over specialisation of rules of adaptation is avoided by deleting empty groups but old rules of adaptation may "survive" to a group deletion by spreading in others groups set of profiles. This case is shown on 6 with the group grp $p_{1}$ which at $t_{9}$ merge it's profile with rules comming from the grp $_{1}$ deleted after $t_{6}$. Although mainly illustrative, this example shows a tentative testbed with users acting and collaborating in a given environment, and adapting a service to their needs and usage.

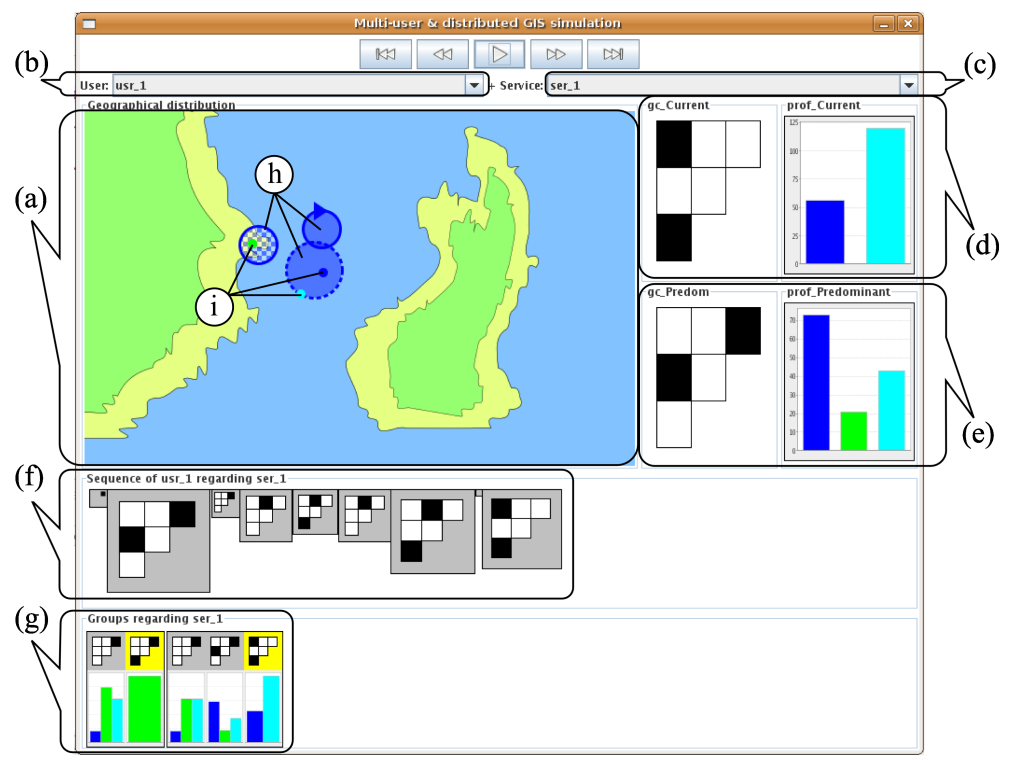

Fig. 7. Simulation prototype interface

\section{Conclusion}

The development of collaborative and adaptive GIS is an area that is becoming crucial for the successful development of many multi-user and location-based applications. Several contextual components such as the characteristics of the users and appliances, and the extent of the geographical environment of interest constitute the elementary constraints of such systems. Although collaborative and adaptive GIS have been already considered by recent research, these 
components are generally considered separatly. The research presented in this paper proposes a contextual approach that takes into account different levels for the processing and display of geographical data in mobile environments. The approach favours the development of an adaptive system, and supports derivation and management of groups and user profiles that form the collaboration dimension of the framework. The collaborative process is driven by the geographical context configurations. Sequences of contextual configurations favour the management of group profiles and allows distribution and sharing of predominant experiences through the distributed GIS. The architecture proposed provides a dynamic structural component to the developement of collaborative GIS environments, where users ans services are likely to interact in different ways and from different motivations. Our approach is illustrated in the context of maritime navigation.

Future work concerns integration of multiple services within the model, and appliance context to refine the user-centered design of the interfaces. We also plan to generate group profiles based on the integration of individual behaviours regarding geographical data and interfaces. Finally, an extension of the approach to other application domains is still to explore, particularly in relationship with the ontologies behind them. 


\section{A Construction of the set of group profiles}

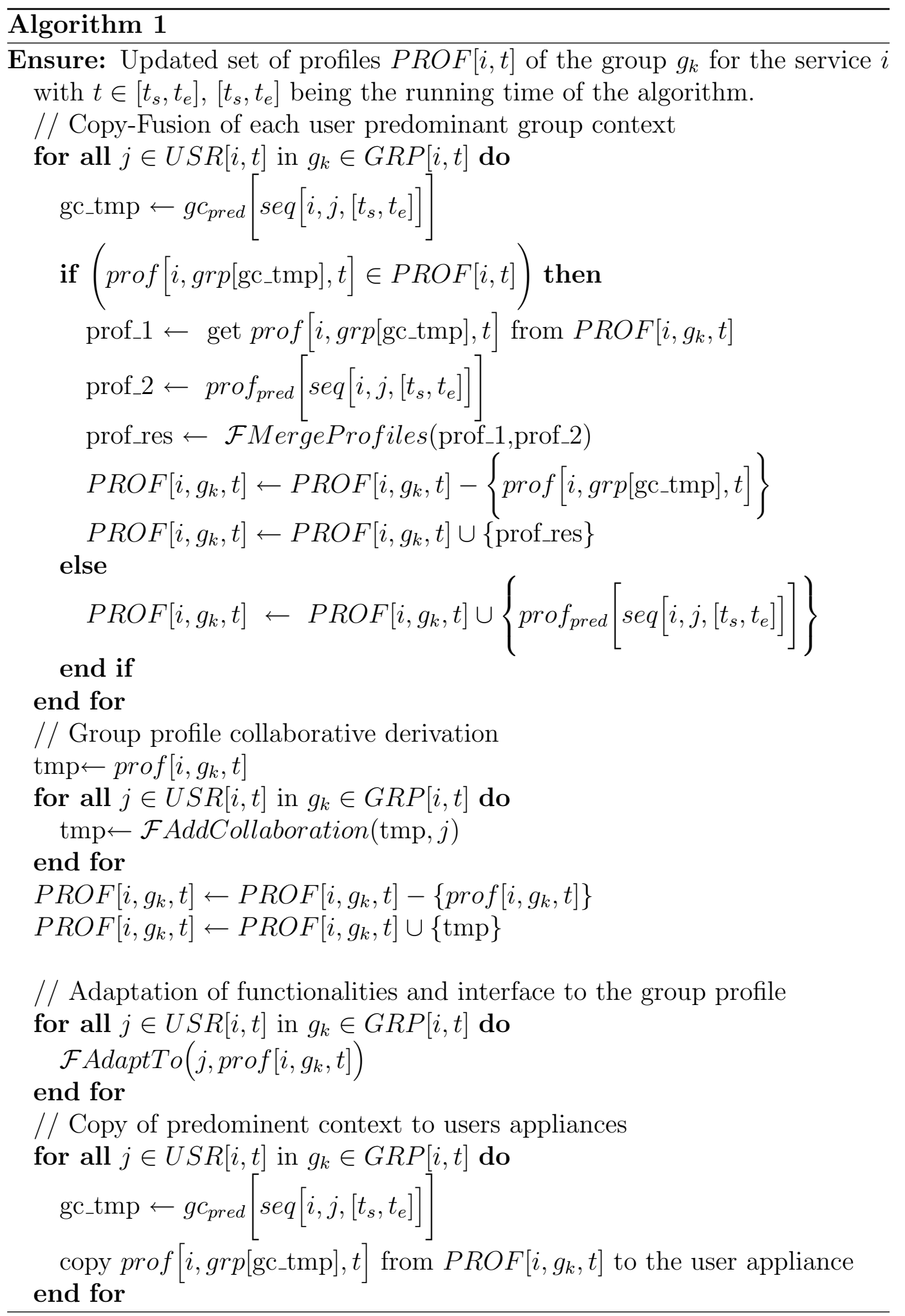




\section{References}

Brown, B., Chalmers, M., MacColl, I., Sep. 2002. Exploring Tourism as A Collaborative Activity. Tech. rep., Equator-02-018.

Burigat, S., Chittaro, L., 2005. Visualizing the results of interactive queries for geographic data on mobile devices. Proceedings of the 13th annual ACM international workshop on Geographic information systems: GIS'05, 277284.

Calvary, G., Coutaz, J., Thevenin, D., Limbourg, Q., Bouillon, L., Vanderdonckt, J., 2003. A unifying reference framework for multi-target user interfaces. Interacting with Computers 15 (3), 289-308.

Desvignes, G., Lucas de Couville, G., Peytchev, E., Devogele, T., Fournier, F., Claramunt, C., 2002. The Share-Loc project: a WAP-based maritime location system. In: proceedings of the WISE Workshops. IEEE Computer Society, pp. 88-94.

Dubs, J., Kaufmann, R., 2006. Gestion et visualisation de données provenant d'un AIS. Tech. rep., Naval Academy Research Institute and Université de la Rochelle.

Hampe, M., Paelke, V., 19 Sep. 2005. Adaptive maps for mobile applications. In: Proceedings of the Mobile Maps Workshop at MobileHCI'05.

Lam, W., Mukhopadhyay, S., Mostafa, J., Palakal, M., 1996. Detection of Shifts in User Interests for Personalized Information Filtering. In: Proceedings of the 19th annual international ACM SIGIR conference on Research and development in information retrieval. pp. 317-325.

Lieberman, H., Van Dyke, N., Vivacqua, A., 1999. Let's browse: a collaborative Web browsing agent. In: Proceedings of the 1999 International Conference on Intelligent User Interfaces: IUI'99. pp. 65-68.

Longley, P., Goodchild, M., Maguire, D., Rhind, D., 2005. Geographical Information Systems and Sciences, 2nd Edition. Willey.

MacEachren, A. M., Cai, G., 2006. Supporting Group Work in Crisis Management: Visually mediated human-GIS-human dialogue. Environment and Planning B: Planning and Design 33 (3), 435-456.

Oard, D., Kim, J., 2001. Modeling information content using observable behavior. In: Proceedings of the 64 Annual Meeting of the American Society for Information Science and Technology. pp. 38-45.

Petit, M., Ray, C., Claramunt, C., Dec. 2006. A contextual approach for the development of GIS: Application to maritime navigation. In: Carswell, J., Tekuza, T. (Eds.), Proceedings of the 6th International Symposium on Web and Wireless Geographical Information Systems: W2GIS'06, to appear. Springer-Verlag LNCS 4295.

Rauschert, I., Agrawal, P., Sharma, R., Fuhrmann, S., Brewer, I., MacEachren, A., 2002. Designing a human-centered, multimodal gis interface to support emergency management. In: Proceedings of the 10th ACM International Symposium on Advances in Geographic Information Systems: Gis'02. ACM Press, New York, NY, USA, pp. 119-124. 
Reichenbacher, T., 2003. Adaptive Methods for Mobile Cartography. In: Proceedings of the 21st International Cartographic Conference: ICC'03. pp. $1311-1322$.

Riecken, D., 2000. Introduction: personalized views of personalization. Communication of the ACM 43 (8), 27-28.

Schafer, J., Konstan, J., Riedl, J., 1999. Recommender systems in e-commerce. In: Proceedings of the ACM Conference on Electronic Commerce. pp. 158166.

Schein, A. I., Popescul, A., Ungar, L. H., 2002. Methods and Metrics for ColdStart Recommendations. In: Proceedings of the 25th annual International ACM SIGIR Conference on Research and Development in Information Retrieval. pp. 253-260.

Shearin, S., Lieberman, H., 2001. Intelligent profiling by example. In: Proceedings of the International Conference on Intelligent User Interfaces: IUI'01. pp. $145-152$.

Weakliam, J., Bertolotto, M., Wilson, D., 2005. Implicit interaction profiling for recommending spatial content. In: Proceedings of the 13th annual ACM international workshop on Geographic information systems: GIS'05. pp. 285-294.

Yang, Y., Claramunt, C., Dec. 2005. A hybrid approach for spatial web personalisation. In: Proceedings of the 5th international workshop on Web and wireless geographical information systems: W2GIS'05. Vol. 3833. pp. 206221.

Zipf, A., 2005. Using styled layer descriptor (SLD) for the dynamic generation of user- and context-adaptative mobile maps - a technical framework. In: Li, K., Vangenot, C. (Eds.), Proceedings of the 5th International Workshop on Web and Wireless GIS (W2GIS 2005). Springer-Verlag, pp. 183-193. 